\title{
Noncrossing Trees and Noncrossing Graphs
}

\author{
William Y. C. Chen and Sherry H. F. Yan \\ Center for Combinatorics, LPMC, Nankai University, 300071 Tianjin, P.R. China \\ chen@nankai.edu.cn, huifangyan@eyou.com
}

Submitted: Sep 18, 2005; Accepted: Nov 30, 2005; Published: Aug 14, 2006

Mathematics Subject Classifications: 05A05, 05C30

\begin{abstract}
We give a parity reversing involution on noncrossing trees that leads to a combinatorial interpretation of a formula on noncrossing trees and symmetric ternary trees in answer to a problem proposed by Hough. We use the representation of Panholzer and Prodinger for noncrossing trees and find a correspondence between a class of noncrossing trees, called proper noncrossing trees, and the set of symmetric ternary trees. The second result of this paper is a parity reversing involution on connected noncrossing graphs which leads to a relation between the number of noncrossing trees with $n$ edges and $k$ descents and the number of connected noncrossing graphs with $n+1$ vertices and $m$ edges.
\end{abstract}

\section{Introduction}

A noncrossing graph with $n$ vertices is a graph drawn on $n$ points numbered in counterclockwise order on a circle such that the edges lie entirely within the circle and do not cross each other. Noncrossing trees have been studied by Deutsch, Feretic and Noy [2], Deutsch and Noy [3], Flajolet and Noy [4], Noy [6], Panholzer and Prodinger [7]. It is well known that the number of noncrossing trees with $n$ edges equals the generalized Catalan number $c_{n}=\frac{1}{2 n+1}\left(\begin{array}{c}3 n \\ n\end{array}\right)$.

In this paper we are concerned with rooted noncrossing trees. We assume that 1 is always the root. A descent is an edge $(i, j)$ such that $i>j$ and $i$ is on the path from the root 1 to the vertex $j$. A ternary tree is either a single node, called the root, or it is a root associated with three ternary trees. A symmetric ternary tree is a ternary tree which can be decomposed into a ternary left subtree, a central symmetric ternary tree and a ternary right subtree that is a reflection of the left subtree, as shown in Figure 1.

Let $\mathcal{S}_{n}$ be the set of symmetric ternary trees with $n$ internal vertices. A noncrossing tree is called even if the number of descents is even. Otherwise, it is called odd. Denote 


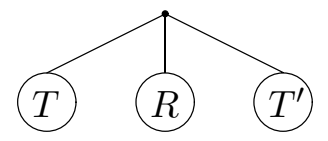

Figure 1: $T^{\prime}$ is the reflection of $T$ and $R$ is symmetric.

by $\mathcal{E}_{n}$ and $\mathcal{O}_{n}$ the sets of even and odd noncrossing trees with $n$ edges, respectively. Let $s_{n}, e_{n}, o_{n}$ be the cardinalities of the sets $\mathcal{S}_{n}, \mathcal{E}_{n}, \mathcal{O}_{n}$, respectively. Deutsch, Feretic and Noy [2] have shown that

$$
s_{n}= \begin{cases}\frac{1}{2 m+1}\left(\begin{array}{c}
3 m \\
m
\end{array}\right) & \text { if } n=2 m, \\
\frac{1}{2 m+1}\left(\begin{array}{c}
3 m+1 \\
m+1
\end{array}\right) & \text { if } n=2 m+1\end{cases}
$$

Recently, Hough [5] obtained the generating function for the number of noncrossing trees with $n$ edges and a prescribed number of descents. He also derived the following relation:

$$
e_{n}-o_{n}=s_{n}
$$

Hough [5] asked the natural question of finding a combinatorial interpretation of the above identity (1.2). In this paper, we obtain a parity reversing involution on noncrossing trees that leads to a combinatorial interpretation of (1.2).

Our combinatorial interpretation of (1.2) relies on the representation of noncrossing trees introduced by Panholzer and Prodinger [7]. Given a noncrossing tree $T$, we may represent it by a plane tree with each vertex labeled by $L$ or $R$ with the additional requirement that the root is not labeled, and the children of the root are labeled by $R$. Such a $(L, R)$-labeled tree representation of $T$ is obtained from $T$ (as a rooted tree) by the following rule: Given any non-root vertex $j$ of $T$, suppose that $i$ is the parent of $j$. If $i>j$ then the label of the vertex corresponding to $j$ is labeled by $L$; otherwise, it is labeled by $R$. These two equivalent representations of noncrossing trees are illustrated by Figure 2. It is obvious that a descent in the noncrossing tree in the first representation corresponds to a $L$-labeled vertex in the second representation.

The second result of this paper is an expression of the number of noncrossing trees with $n$ edges and $k$ descents in terms of the number of connected noncrossing graphs with $n+1$ vertices and $k$ edges. Noncrossing graphs have been extensively studied by Flajolet and Noy [4]. They derived the following formula for the number of connected noncrossing graphs with $n+1$ vertices and $k$ edges, that is,

$$
N_{n, k}=\frac{1}{n}\left(\begin{array}{c}
3 n \\
n+1+k
\end{array}\right)\left(\begin{array}{l}
k-1 \\
n-1
\end{array}\right) .
$$



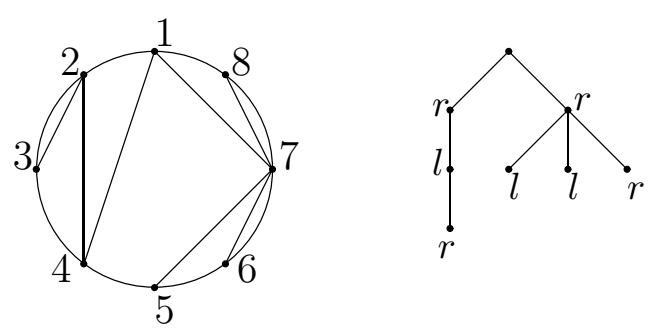

Figure 2: Two representations of a noncrossing tree

Hough [5] found a combinatorial interpretation of the relation between the descent generating function of noncrossing trees and the generating function for connected noncrossing graphs. By using the Lagrange inversion formula he obtained the following explicit formula for the number of noncrossing trees with $n$ edges and $k$ descents,

$$
d_{n, k}=\frac{1}{n}\left(\begin{array}{c}
n-1+k \\
n-1
\end{array}\right)\left(\begin{array}{c}
2 n-k \\
n+1
\end{array}\right)
$$

As the second result of this paper, we present a parity reversing involution on connected noncrossing graphs and obtain an expression for the number $d_{n, k}$ in terms of the numbers $N_{n, m}$.

\section{An involution on noncrossing trees}

In this section, we give a parity reversing involution on noncrossing trees which leads to a combinatorial interpretation of the relation (1.2). We use the representation of noncrossing trees introduced by Panholzer and Prodinger [7]. Let $T$ be an even noncrossing tree with $n$ edges and $v$ be a non-root internal node of $T$. A vertex $v$ is called a proper vertex if it has an even number of left children but has no right child. If $T$ is odd, that is, $T$ has an odd number of descents, then $v$ is said to be proper if $v$ has an even number of right children but has no left child. Otherwise, $v$ is said to be improper. A noncrossing tree is said to be proper if every non-root vertex is proper. Otherwise, it is said to be improper. It is obvious that each odd noncrossing tree is improper. Let us use $\mathcal{T}_{n}$ to denote the set of proper noncrossing trees with $n$ internal nodes and let $t_{n}$ denote the cardinality of $\mathcal{T}_{n}$.

Let us recall that a plane tree is said to be an even tree if each vertex has an even number of children. Chen [1] gives a bijection $\psi$ between the set of even plane trees with $2 n$ edges and the set of ternary trees with $n$ internal nodes. A similar bijection is obtained by Deutsch, Feretic and Noy [2]. Here we give a brief description of this bijection. Suppose that $T$ is an even plane tree with $2 n$ edges. We use the following procedure to construct a ternary tree with $n$ internal vertices.

- Step 1. Construct two plane trees $T_{1}$ and $T_{2}$ based on $T: T_{1}$ is the subtree containing 
the root and the first two subtrees of $T$, whereas $T_{2}$ is the subtree of $T$ obtained by removing the first two subtrees of the root.

- Step 2. Combine $T_{2}$ with $T_{1}$ by joining $T_{2}$ as the last subtree of the root of $T_{1}$.

- Step 3. Repeat the above procedure for all the nontrivial subtrees (with at least two vertices) of the root.

Since each non-root vertex of a proper even noncrossing tree has only an even number of left children and has no right child, we can discard the labels of its children and represent a proper tree as a plane tree such that each subtree of the root is an even tree. We define a map $\sigma: \mathcal{T}_{n} \rightarrow \mathcal{S}_{n}$ as follows.

The map $\sigma$ : Let $T$ be a proper even noncrossing tree. Let $T_{1}$ be the first subtree of the root. The map is defined by a recursive procedure.

- Step 1. Assign a vertex as the root and let $\psi\left(T_{1}\right)$ be the first subtree of the root and its reflection be the third subtree of the root.

- Step 2. Let $T_{2}$ be the subtree obtained from $T$ by deleting $T_{1}$, and let $\sigma\left(T_{2}\right)$ be the second subtree of the root.

The above map $\sigma$ is clearly a bijection between $\mathcal{T}_{n}$ and $\mathcal{S}_{n}$. Figure 3 is an example.

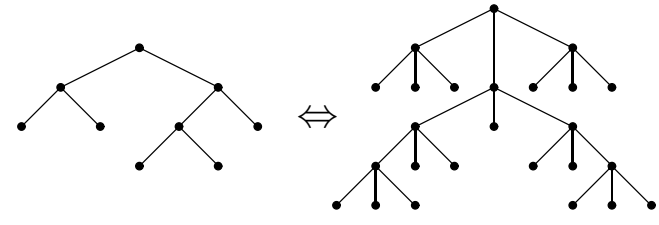

Figure 3: The map $\sigma$

Theorem 2.1 The map $\sigma$ is a bijection between the set of proper noncrossing trees with $n$ edges and the set of symmetric ternary trees with $n$ internal vertices.

By using even plane trees as an intermediate structure, we may obtain a combinatorial interpretation of (1.2) by constructing an involution on improper noncrossing trees which changes the parity of the number of descents.

Theorem 2.2 There is a parity reversing involution on the set of improper noncrossing trees with $n$ edges. So we have the following relation

$$
e_{n}-o_{n}=t_{n}
$$


Proof. Let $T$ be an improper noncrossing tree with $n$ edges. Traverse $T$ in preorder and let $v$ be the first encountered improper node. Define the map $\phi$ as follows: Case (1), if $T$ is an odd tree and $v$ has at least one left child, then $\phi(T)$ is obtained by changing its rightmost left child to a right child and changing all the children of the non-root vertices traversed before $v$ to left children; Case (2), if $T$ is an odd tree and $v$ has no left children but has an odd number of right children, then $\phi(T)$ is obtained by changing all the children of $v$ to left children and changing all the children of non-root nodes traversed before $v$ to left children.

If $T$ is an even tree and $v$ has at least one right child, then one can reverse the construction in Case (1). If $T$ is an even tree and $v$ has no right child and has an odd number of left children, then the construction in Case (2) is also reversible. Hence the map $\phi$ is an involution on the set of improper noncrossing trees with $n$ edges. Moreover, one sees that this involution changes the parity of the number of descents. Thus, we obtain the relation (2.1).

An example of the above involution is illustrated in Figure 4.

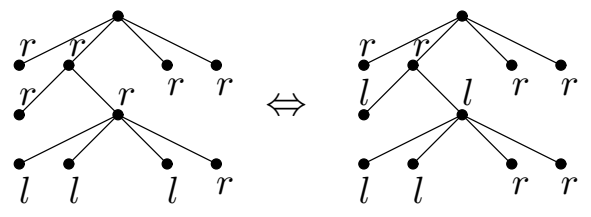

Figure 4: The involution $\phi$

Combining the bijections in Theorems 2.1 and 2.2, we get a combinatorial interpretation of the relation (1.2). Note that equation (1.2) leads to the following two combinatorial identities

$$
\begin{aligned}
\sum_{k=0}^{2 m-1}(-1)^{k}\left(\begin{array}{c}
2 m-1+k \\
k
\end{array}\right)\left(\begin{array}{c}
4 m-k \\
2 m+1
\end{array}\right) & =\frac{2 m}{2 m+1}\left(\begin{array}{c}
3 m \\
m
\end{array}\right) \\
\sum_{k=0}^{2 m}(-1)^{k}\left(\begin{array}{c}
2 m+k \\
k
\end{array}\right)\left(\begin{array}{c}
4 m+2-k \\
2 m+2
\end{array}\right) & =\left(\begin{array}{c}
3 m+1 \\
m+1
\end{array}\right) .
\end{aligned}
$$

\section{An involution on connected noncrossing graphs}

In this section, we aim to establish a connection between the number of noncrossing trees with $n$ edges and $k$ descents and the number of connected noncrossing graphs with $n+1$ vertices and $m$ edges. 
Theorem 3.1 We have the following relation

$$
\sum_{m=n}^{2 n-1}(-1)^{m-n}\left(\begin{array}{c}
m-n \\
k
\end{array}\right) N_{n, m}=(-1)^{k} d_{n, k} .
$$

Let $G$ be a connected noncrossing graph with vertex set $\{1,2, \ldots, n+1\}$. We may construct a unique spanning tree of $G$, which is called the canonical spanning tree of $G$. This construction can be viewed as a reformulation of the traversal procedure of Hough [5]. Since $G$ is noncrossing, any cycle of $G$ can be represented by a sequence $\left(i_{1}, i_{2}, \ldots, i_{k}\right)$ such that $i_{1}<i_{2}<\cdots<i_{k}$, and $\left(i_{1}, i_{2}\right),\left(i_{2}, i_{3}\right), \ldots,\left(i_{k-1}, i_{k}\right)$ and $\left(i_{k}, i_{1}\right)$ are the edges of the cycle. For a cycle $\left(i_{1}, i_{2}, \ldots, i_{k}\right)$ represented in the above form, we may delete the edge $\left(i_{1}, i_{2}\right)$ to break the cycle until we obtain a spanning tree. An example is shown in Figure 5. We have the following uniqueness property of the canonical spanning tree.

Proposition 3.2 Let $G$ be a connected noncrossing graph. The canonical spanning tree of $G$ does not depend on the order of the cycles chosen in the edge deletion procedure.

Proof. Suppose that we get two different canonical spanning trees $T$ and $T^{\prime}$ of a connected noncrossing graph $G$ by using different orders of the cycles for the edge deletion procedures. Assume that $\left(i_{1}, i_{2}\right) \notin E(T)$ and $\left(i_{1}, i_{2}\right) \in E\left(T^{\prime}\right)$. Suppose that $C_{1}, C_{2}, \cdots, C_{r}$ and $C_{1}^{\prime}, C_{2}^{\prime}, \cdots, C_{r}^{\prime}$ are the cycles encountered in the edge deletion procedures for $T$ and $T^{\prime}$. Since $\left(i_{1}, i_{2}\right) \notin E(T)$, we may assume that $\left(i_{1}, i_{2}\right) \in E\left(C_{j}\right)$ and $i_{1}$ and $i_{2}$ are the minimum and the second minimum numbers of $C_{j}$. Since $\left(i_{1}, i_{2}\right) \in E\left(T^{\prime}\right)$, we may find the minimum integer $t$ such that after breaking the cycle $C_{t}^{\prime}$ by deleting the appropriate edge, the numbers $i_{1}, i_{2}$ are no longer the minimum and second minimum numbers in the cycles. Let $G_{t}^{\prime}$ be the subgraph of $G$ obtained by the operations of breaking the cycles $C_{1}^{\prime}, C_{2}^{\prime}, \ldots, C_{t-1}^{\prime}$. Let $C=\left(i_{1}, i_{2}, \ldots, i_{k}\right)$ be a cycle in $G_{t}^{\prime}$. Then the cycle $C_{t}^{\prime}$ can be represented as $\left(i_{s}, i_{s+1}, j_{1}, j_{2}, \ldots, j_{p}\right)$ or $\left(i_{1}, i_{k}, j_{1}, j_{2}, \ldots, j_{p}\right)$. In the first case, since the graph $G$ is noncrossing, $j_{1}, j_{2}, \ldots, j_{p}$ are on the cycle $C$. Assume that $j_{p}=i_{q}$. After breaking $C_{t}^{\prime}$, there is also a cycle $\left(i_{1}, i_{2}, \ldots, i_{s}, i_{q}, \ldots, i_{k}\right)$ with $i_{1}, i_{2}$ being the minimum and second minimum numbers. In the second case, after breaking $C_{t}^{\prime}$, there is also a cycle $\left(i_{1}, i_{2}, \ldots, i_{k}, j_{1}, j_{2}, \ldots, j_{p}\right)$ with $i_{1}, i_{2}$ being the minimum and second minimum numbers. Both the above two cases contradict with the assumption for $C_{t}^{\prime}$. Thus $T$ and $T^{\prime}$ are identical.

Conversely, given a noncrossing tree $T$ with $n$ edges and a subset $S$ of its descents, we can construct a connected noncrossing graph by using the bijection of Hough [5] which can be described as follows: For each descent $(i, j)$ in $S$, find the maximal path of consecutive descents from $j$ back to the root, and let the first vertex on this path be $v$. From the neighbors of the vertices on the path from $v$ to $i$ except for the vertices on the path, choose the neighbor $w$ as the largest vertex less than $j$; Then add the new edge $(w, j)$ to $T$. We call the new edge $(w, j)$ the companion edge of the descent $(i, j)$.

An edge in $G$ is said to be free if it is not in the canonical spanning tree $T$. A descent $(i, j)$ in the canonical spanning tree of a connected noncrossing graph is said to be saturated 

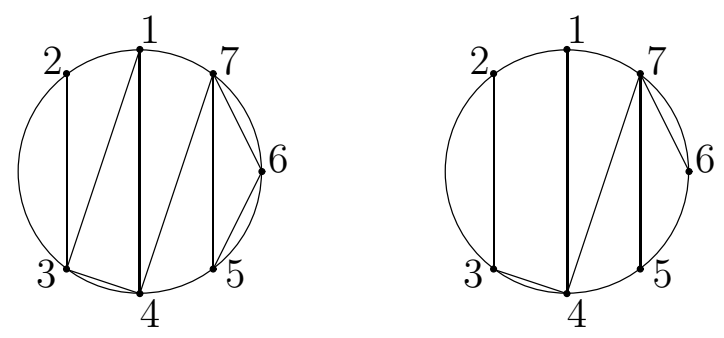

Figure 5: The canonical spanning tree

if its companion edge is contained in the connected noncrossing graph. Otherwise, it is said to be unsaturated.

We now need to consider connected noncrossing graphs in which some of the free edges are marked. Denote by $\mathcal{N}_{n, m, k}$ the set of connected noncrossing graphs with $n+1$ vertices and $m$ edges and $k$ marked free edges. It is clear to see that the cardinality of the set $\mathcal{N}_{n, m, k}$ is given by

$$
\left(\begin{array}{c}
m-n \\
k
\end{array}\right) N_{n, m} .
$$

Denote by $\mathcal{N}_{n, k}$ the set of connected noncrossing graphs with $n+1$ vertices and $k$ marked free edges. A descent $(i, j)$ in the canonical spanning tree of a connected noncrossing graph is said to be marked if its companion edge is marked. Denote by $\mathcal{D}_{n, k}$ the set of connected noncrossing graphs with $n+1$ vertices and $n+k$ edges such that each descent in its spanning tree is marked. It follows that $\left|\mathcal{D}_{n, k}\right|=d_{n, k}$. We will be concerned with the set $\mathcal{N}_{n, k}-\mathcal{D}_{n, k}$, that is, the set of connected noncrossing graphs with $n+1$ vertices and $k$ marked free edges which contain at least one unmarked descent.

Note that two descents $(i, j)$ and $\left(i^{\prime}, j^{\prime}\right)$ can not share an end vertex, namely, $j \neq j^{\prime}$. A descent $(i, j)$ is said to be smaller than a descent $\left(i^{\prime}, j^{\prime}\right)$ if $j<j^{\prime}$. We now give an involution on the set $\mathcal{N}_{n, k}-\mathcal{D}_{n, k}$ that reverses the parity of the number of free edges.

Theorem 3.3 There is an involution on the set $\mathcal{N}_{n, k}-\mathcal{D}_{n, k}$ that reverses the parity of the number of free edges.

Proof. Let $G$ be a connected noncrossing graph in $\mathcal{N}_{n, k}-\mathcal{D}_{n, k}$ with $m-n$ free edges. We define a map $\psi$ as follows. First, find the minimum unmarked descent $(i, j)$. We have two cases. Case 1: The descent $(i, j)$ is saturated in $G$. We delete the companion edge of $(i, j)$ to get a connected noncrossing graph with $n+1$ vertices, $m-n-1$ free edges and $k$ marked free edges. Case 2 : The descent $(i, j)$ is not saturated in $G$. We add the companion edge of $(i, j)$ to get a connected noncrossing graph with $n+1$ vertices, $m-n+1$ free edges and $k$ marked free edges. The operations in the two cases clearly constitute an involution that changes the number of free edges by one.

As a consequence of Theorem 3.3, we obtain the identity (3.1). 
To conclude this paper, we remark that Theorem 3.1 can be deduced from the formulas (1.3) and (1.4) for $N_{n, k}$ and $d_{n, k}$ and the following identity

$$
\sum_{m=n}^{2 n-1}(-1)^{m-n-k}\left(\begin{array}{c}
3 n \\
n+1+m
\end{array}\right)\left(\begin{array}{c}
m-1 \\
n-1
\end{array}\right)\left(\begin{array}{c}
m-n \\
k
\end{array}\right)=\left(\begin{array}{c}
n-1+k \\
n-1
\end{array}\right)\left(\begin{array}{c}
2 n-k \\
n+1
\end{array}\right),
$$

which can be verified by using the Vandermonde convolution [8, p. 8]

$$
\left(\begin{array}{c}
n-m \\
k
\end{array}\right)=\sum_{i+j=k}(-1)^{i}\left(\begin{array}{c}
m+i-1 \\
i
\end{array}\right)\left(\begin{array}{l}
n \\
j
\end{array}\right) .
$$

Acknowledgments. We would like to thank the referee for helpful suggestions. This work was supported by the 973 Project on Mathematical Mechanization, the National Science Foundation, the Ministry of Education, and the Ministry of Science and Technology of China.

\section{References}

[1] W.Y.C. Chen, A general bijective algorthm for increasing trees, Systems Science and Mathematical Sciences, 12 (1999) 194-203.

[2] E. Deutsch, S. Feretic and M. Noy, Diagonally convex directed polyominoes and even trees: a bijection and related issues, Discrete Math. 256 (2002) 645-654.

[3] E. Deutsch and M. Noy, Statistics on non-crossing trees, Discrete Math., 254 (2002) 75-87.

[4] P. Flajolet and M. Noy, Analytic combinatorics of non-crossing configurations, Discrete Math., 204 (1999) 203-229.

[5] D.S. Hough, Descents in noncrossing trees, Electronic J. Combin., 10 (2003) N13.

[6] M. Noy, Enumeration of noncrossing trees on a circle, Discrete Math., 180 (1998) 301-313.

[7] A. Panholzer and H. Prodinger, Bijections for ternary trees and noncrossing trees, Discrete Math., 250 (2002) 181-195.

[8] J. Riordan, Combinatorial Identities, John Wiley \& Sons, Inc., 1968. 